\title{
NONEXISTENCE AND UNIQUENESS OF POSITIVE SOLUTIONS OF YAMABE TYPE EQUATIONS ON NONPOSITIVELY CURVED MANIFOLDS
}

\author{
BRUNO BIANCHINI AND MARCO RIGOLI
}

\begin{abstract}
We prove nonexistence and uniqueness of positive $C^{2}$-solutions of the elliptic equation $\Delta u+a(x) u-K(x) u^{\sigma}=0, \quad \sigma>1$, on a nonpositively curved, complete manifold $(M, g)$.
\end{abstract}

\section{INTRODUCTION}

Let $(M, g)$ be a complete, noncompact Riemannian manifold of dimension $m \geq$ 3 and let $a(x), K(x)$ be assigned continuous functions on $M$. The aim of this paper is to determine conditions, involving the asymptotic behavior of $a, K$ and the geometry of $(M, g)$, in order to guarantee nonexistence, and eventually uniqueness, of positive $C^{2}$-solutions of the elliptic equation

$$
\Delta u+a(x) u-K(x) u^{\sigma}=0
$$

on $M$, for some constant $\sigma>1$. This problem has geometrical roots. Indeed, up to an inessential multiplicative positive constant in front of $\Delta$, the choice $\sigma=\frac{m+2}{m-2}$ in (0.1) produces the well-known Yamabe equation relating the scalar curvature $-a(x)$ of $g$ to that, $-K(x)$, of the conformally deformed metric $g_{u}=u^{\frac{4}{m-2}} g, u>0$. In this case a fairly accurate general existence result, satisfying the further request of completeness of $g_{u}$, has been given in [RRV1] for "negative" scalar curvatures.

In the present investigation, as naturally expected on a Riemannian manifold, the geometry is controlled via curvature conditions. To be more precise we have fixed our attention on the two most typical cases

$$
\text { i) } \operatorname{Riem}_{(M, g)} \leq 0 \text { and ii) } \operatorname{Riem}_{(M, g)} \leq-B^{2}
$$

for some constant $B>0$. Here $\operatorname{Riem}_{(M, g)}$ denotes the sectional curvature. Thus, $R^{m}$ with its canonical metric pertains to case i) and, in this special setting, under the further assumption $a(x) \equiv 0$, a number of results have been obtained, for instance, by $\mathrm{Ni}[\mathrm{N}]$, Lin $[\mathrm{L}]$, and more recently by Cheng and Lin $[\mathrm{C}-\mathrm{L}]$. However, our methods, beside having a geometrical flavor, differ from those of the above authors, mainly because, while in $R^{m}$ standard symmetrization techniques are at hand, on a general manifold such an approach is obviously not feasible. Furthermore, the appearance of the linear term in (0.1) plays a role, in connection with $K(x)$, which, to our knowledge, has not been thoroughly analysed even in euclidean space. As

Received by the editors March 6, 1995.

1991 Mathematics Subject Classification. Primary 53C21, 58G03.

Key words and phrases. Maximum principles, elliptic differential inequalities, Riemannian geometry. 
a matter of fact, our results complement Theorems 2.1, 2.2 and 2.3 of [C-L]. (See Corollary 2.4 below.) To give a sample of the type of conclusions that we obtain, we report a version of our main result under assumption i). A slightly more general statement is contained in Theorems 2.1 of section 2 .

From now on, $(M, g)$ shall always be assumed to be connected, simply connected, complete and of dimension $m \geq 3$ unless otherwise specified. Having fixed $p \in M$, we set

$$
r(x)=\operatorname{dist}_{(M, g)}(p, x) .
$$

The choice of $p$ plays no significant role.

Theorem A. Let $(M, g)$ satisfy $i)$ and $a(x), K(x) \in C^{o}(M)$. Assume that

$$
\begin{aligned}
a(x) & \leq \frac{A}{r(x)^{2}} \quad \text { on } M \text { for some } A \leq \frac{(m-2)^{2}}{4}, \\
K(x) & \geq 0 \quad \text { on } M,
\end{aligned}
$$

there exist constants $c>0, \sigma>1$ such that

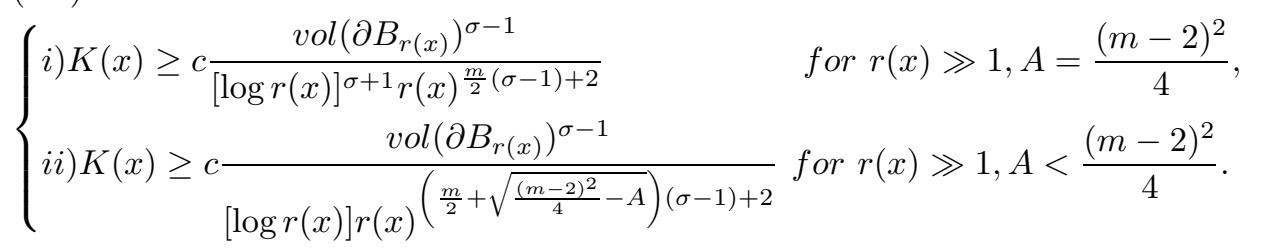

Then, the differential inequality

$$
\Delta u+a(x) u \geq K(x) u^{\sigma}
$$

has no positive $C^{2}$-solution on $M$.

(For a discussion on assumptions (0.2) and (0.4) in case $A \geq 0$ see section 2.)

In case ii) our conclusions resemble those obtained in i). However, a comparison of the two cases clarifies the role of sectional curvature. As an interesting geometrical consequence, regarding the Yamabe equation, we obtain:

Corollary B. Let $(M, g)$ satisfy ii) and let $K(x) \in C^{\infty}(M)$ be such that

$$
K(x) \leq-c \frac{\operatorname{vol}\left(\partial B_{r(x)}\right)^{\frac{4}{(m-2)}}}{r(x) \log r(x) e^{\frac{2 m}{m-2} B r(x)}} \quad \text { for } r(x) \gg 1
$$

and some constant $c>0 . \quad$ Assume that the scalar curvature $s(x)$ of $(M, g)$ satisfies

$$
s(x) \geq-m(m-1) B^{2} \operatorname{coth} B r(x) .
$$

Then, if $K(x) \leq 0$ on $M$, the metric $g$ cannot be conformally deformed to a metric of scalar curvature $K(x)$.

As a side product of our qualitative study, we find estimates from below for the spherical mean of nonnegative solutions of the differential inequality

$$
\Delta u+a(x) u \geq 0 .
$$

These, together with a technique developed in [RRV1], enable us to obtain a uniqueness result for positive solutions of (0.1), that is, 
Theorem C. Let $(M, g)$ be such that

$$
\operatorname{Riem}_{(M, g)} \leq-B^{2}
$$

for some $B \geq 0$. Let $a(x), K(x) \in C^{0}(M)$ and assume

$$
a(x) \leq \begin{cases}\frac{(m-2)^{2}}{4} \frac{1}{r(x)^{2}} & \text { in case } B=0, \\ \frac{(m-1)^{2}}{4} B^{2} \operatorname{coth} B r(x) & \text { in case } B>0 .\end{cases}
$$

on $M$;

$$
\text { i) } K(x) \geq 0 ; \quad \text { ii) } \quad K(x) \not \equiv 0 \quad \text { on } M .
$$

Let $u, v \in C^{2}(M)$ be positive solutions of (0.1) for some $\sigma>1$. If

$$
(u-v)(x)=o\left(\left\{\begin{array}{lll}
r(x)^{-\frac{m-2}{2}} \log r(x) & \text { in case } & B=0 \\
r(x) e^{-\frac{m-1}{2} B r(x)} & \text { in case } & B>0
\end{array}\right) \text { as } r(x) \rightarrow+\infty,\right.
$$

then $u \equiv v$ on $M$.

The paper is organized as follows. In section 1 we fix notation and collect a number of technical results that are essential in the subsequent paragraphs. Sections 2 and 3 deal, respectively, with cases i) and ii). In section 4 we prove Theorem $\mathrm{C}$ and some further consequences of our analysis.

We are grateful to the referee for having brought to our attention a paper by Gui and Wang (The critical asymptotics of scalar curvatures of the conformal complete metrics with negative curvature. Duke Math. J. 76 (1994), 293-302) which contains a nonexistence result for the Yamabe problem related to our Theorem 3.1 below.

\section{Preliminary Results}

In this section we fix notations and collect a number of technical lemmas which will be essential in establishing our results. However, on a first reading, it is probably convenient to skip proofs. We begin with a result motivated by the work of Cheng and Lin, [C-L].

Lemma 1.1. Let $0<R_{0} \leq R_{1} \leq R_{2} \leq+\infty, \sigma>1$ and assume that the functions $l, \Psi, b \in C^{0}\left(\left[R_{0},+\infty\right)\right)$ satisfy

$$
\begin{cases}\left.i) \quad l(t) \geq 0 \quad \text { on }\left[R_{0},+\infty\right) ; \quad i i\right) \quad \Psi(t)>0 \quad \text { on }\left[R_{0},+\infty\right) \\ \left.i i i) \quad t b(t) \notin L^{1}(+\infty) ; \quad i v\right) \quad t b(t)>0 \quad \text { and nonincreasing on }\left[R_{1},+\infty\right) .\end{cases}
$$

Suppose that

$$
\liminf _{t \rightarrow \infty} \frac{l(t) \Psi(t)^{\sigma}}{t b(t)}>0
$$

If $\alpha$ is a positive solution of

$$
\alpha(s) \geq \Psi(s)\left\{c_{1}+c_{2} \int_{R_{0}}^{s}\left(1-\frac{t}{s}\right) l(t) \alpha(t)^{\sigma} d t\right\}
$$

on $\left[R_{0}, R_{2}\right)$, for some positive constants $c_{1}, c_{2}$, then $R_{2}<+\infty$. 
Remark 1.4. i) In Lemma 1.1 we can relax both (1.3) to

$$
\alpha(s) \geq \Psi(s)\left\{c_{1}+c_{2} \int_{R_{0}}^{s}\left[1-\left(\frac{t}{s}\right)^{a}\right] l(t) \alpha(t)^{\sigma} d t\right\}
$$

on $\left[R_{0}, R_{2}\right)$, for some positive constants $a, c_{1}, c_{2}$; and (1.1)iv) to

$(1.1) v)$

$$
\left\{\begin{array}{l}
\int_{R_{1}}^{t} s b(s) d s \quad \text { is strictly increasing and there exists } \eta \in(0,1] \text { such that } \\
\liminf _{t \rightarrow+\infty} \int_{R_{1}}^{t} s b(s) d s-\eta t^{2} b(t)>-\infty .
\end{array}\right.
$$

We shall make use of (1.1) $v$ ) in Corollary 2.5.

ii) Examples of functions $b(t)$ satisfying (1.1) iii) and $i v$ ), for $t$ sufficiently large, are

$$
b_{1}(t)=t^{-2} ; \quad b_{2}(t)=t^{-2}(\log t)^{-1} ; \quad b_{3}(t)=t^{-2}(\log t \log \log t)^{-1}
$$

and so on. We observe that when $b(t)=b_{n}(t)$ for some $n \in N, n \geq 1$, the conclusion of Lemma 1.1 holds true even for a positive solution $\alpha$ of

$$
\alpha(s) \geq \Psi(s)\left\{c_{1}+c_{2} \int_{R_{0}}^{s}\left(1-\frac{\log t}{\log s}\right) l(t) \alpha(t)^{\sigma} d t\right\}
$$

on $\left[R_{0}, R_{2}\right)$.

iii) The proof of the above assertions is a simple modification of the argument presented below. We have decided to limit ourselves to the case contained in Lemma 1.1 for the sake of simplicity.

Proof (of Lemma 1.1). We argue by contradiction and let $R_{2}=+\infty$. We set

$$
\beta(s)=\frac{\alpha(s)}{\Psi(s)}
$$

so that (1.3) implies that $\beta$ satisfies

$$
\beta(s) \geq c_{1}+c_{2} \int_{R_{0}}^{s}\left(1-\frac{t}{s}\right) l(t) \Psi(t)^{\sigma} \beta(t)^{\sigma} d t \quad \text { for each } \quad s \geq R_{0} .
$$

Because of (1.2), there exist $c_{3}>0$ and $R_{3} \geq R_{0}$ such that

$$
\beta(s) \geq c_{1}+c_{3} \int_{R}^{s}\left(1-\frac{t}{s}\right) t b(t) \beta(t)^{\sigma} d t \quad \text { for each } s \geq R \geq R_{3} .
$$

We choose $R_{4}>\max \left\{R_{1}, R_{3}\right\}$ and we define

$$
\eta(t)=\int_{R_{4}}^{t} v b(v) d v+R_{4}^{2} b\left(R_{4}\right)
$$

We observe that

$$
\eta(t) \geq 0
$$

and

$$
\begin{cases}\text { i) } & \eta^{\prime}(t)=t b(t)>0 \\ \text { ii) } & \eta(t) \rightarrow+\infty \quad \text { as } t \rightarrow+\infty\end{cases}
$$


because of assumptions (1.1) iii), iv). We let $R \geq R_{4}$ and we consider

$$
\eta: \quad[R, s] \rightarrow[\nu=\eta(R), \xi=\eta(s)] .
$$

We indicate with $t(\eta)$ its inverse. We set

$$
\gamma(\eta)=\beta(t(\eta))
$$

and we perform a change of variable in (1.6) to obtain

$$
\gamma(\xi) \geq c_{1}+c_{3} \int_{\nu}^{\xi}\left[1-\frac{t(\eta)}{t(\xi)}\right] \gamma(\eta)^{\sigma} d \eta
$$

Next, we observe that for $\eta$ sufficiently large, say $\eta \geq \eta_{0}, \frac{t(\eta)}{\eta}$ is nondecreasing. Indeed, it is enough to show that

$$
\eta t^{\prime}(\eta)-t(\eta) \geq 0, \quad \eta \geq \eta_{0}
$$

This, in turn, is equivalent to showing that

$$
\int_{R_{4}}^{t} v b(v) d v+R_{4}^{2} b\left(R_{4}\right)-t^{2} b(t) \geq 0
$$

for $t$ sufficiently large. But (1.1) iv) and our choice of $R_{4}$ imply (1.10) for $t \geq R_{4}$. From (1.9) we then deduce

$$
\gamma(\xi) \geq c_{1}+c_{3} \int_{\nu}^{\xi}\left(1-\frac{\eta}{\xi}\right) \gamma(\eta)^{\sigma} d \eta
$$

for each $\xi>\nu \geq \eta\left(R_{4}\right)$. With the aid of (1.8) ii) and (1.11) we then have: for each $\nu \geq \eta\left(R_{4}\right)$ and each $\xi \in[\nu, 2 \nu]$,

$$
\gamma(\xi) \geq c_{1}+c_{3} \frac{1}{2 \nu} \int_{\nu}^{\xi}(\xi-\eta) \gamma(\eta)^{\sigma} d \eta
$$

To complete the proof we imitate the final part of the argument in Theorem 2.1 of $[\mathrm{C}-\mathrm{L}]$. For the sake of completeness we reproduce the reasoning here. We define

$$
g(\xi)=c_{1}+c_{3} \frac{1}{2 \nu} \int_{\nu}^{\xi}(\xi-\eta) \gamma(\eta)^{\sigma} d \eta
$$

and we observe that $g$ satisfies

$$
\left\{\begin{array}{l}
\text { i) } g(\nu)=c_{1}>0 ; \quad \text { ii) } g^{\prime}(\nu)=0 \\
\text { iii } \left.) \quad g^{\prime}(\xi)=\frac{c_{3}}{2 \nu} \int_{\nu}^{\xi} \gamma(\eta)^{\sigma} d \eta ; \quad \text { iv }\right) g^{\prime \prime}(\xi)=\frac{c_{3}}{2 \nu} \gamma(\xi)^{\sigma} \geq \frac{c_{3}}{2 \nu} g(\xi)^{\sigma}
\end{array}\right.
$$

where in (1.14) iv) we have been using (1.12) and (1.13). From (1.14) we obtain

$$
\left(\left[g^{\prime}(\xi)\right]^{2}\right)^{\prime} \geq \frac{c_{3}}{(\sigma+1) \nu}\left(g(\xi)^{\sigma+1}\right)^{\prime} .
$$

Therefore, integrating (1.15) over $[\nu, \xi]$, with the aid of (1.14)ii) we deduce

$$
g^{\prime}(\xi) \geq\left[\frac{c_{3}}{(\sigma+1) \nu}\right]^{\frac{1}{2}}\left[g(\xi)^{\sigma+1}-g(\nu)^{\sigma+1}\right]^{\frac{1}{2}} .
$$

Integrating (1.16), again over $[\nu, \xi]$, and using (1.14) iii) we have

$$
\int_{\nu}^{\xi} \frac{g^{\prime}(x)}{\left[g(x)^{\sigma+1}-g(\nu)^{\sigma+1}\right]^{\frac{1}{2}}} d x \geq\left[\frac{c_{3}}{(\sigma+1) \nu}\right]^{\frac{1}{2}}(\xi-\nu) .
$$


Next, we perform the change of variable

$$
u=\frac{g(x)}{g(\nu)}
$$

and we rewrite (1.17) in the form

$$
\int_{1}^{\frac{g(\xi)}{c_{1}}} \frac{d u}{\left(u^{\sigma+1}-1\right)^{\frac{1}{2}}} \geq\left[\frac{c_{1} c_{3}}{(\sigma+1) \nu}\right]^{\frac{1}{2}}(\xi-\nu) .
$$

But $\sigma>1$; hence $\left(u^{\sigma+1}-1\right)^{-\frac{1}{2}} \in L^{1}([1,+\infty))$. On the other hand, choosing $\xi=2 \nu$ in (1.18), we immediately obtain a contradiction by taking $\nu$ sufficiently large.

To state the next result, we introduce some notation that shall be repeatedly used in the sequel. Given $\sigma>1$ and a nonnegative $f(x) \in C^{o}(M)$ we set:

$$
\begin{aligned}
& \bar{f}(r)=\frac{1}{\operatorname{vol}\left(\partial B_{r}(p)\right)} \int_{\partial B_{r}(p)} f, \quad r \in[0,+\infty), \\
& \bar{f}_{\sigma}(r)=\left\{\frac{1}{\operatorname{vol}\left(\partial B_{r}(p)\right)} \int_{\partial B_{r}(p)} f^{\frac{1}{1-\sigma}}\right\}^{1-\sigma}, \quad r \in[0,+\infty),
\end{aligned}
$$

respectively for the spherical mean and the weighted spherical mean of $f$, with the convention that $\bar{f}_{\sigma}(r)=0$ in case the integral in (1.20) is infinite.

Lemma 1.2. Let $(M, g)$ be such that $m \geq 2$ and assume

$$
\Delta r \geq(m-1) z(r)
$$

for some $z(r) \in C^{o}([0,+\infty))$ in the weak sense on $M$. Let $a(x), K(x) \in C^{o}(M)$ satisfy

$$
a(x) \leq p(r(x)) \quad \text { on } M /\{p\}
$$

for some $p(t) \in C^{o}((0,+\infty))$,

$$
K(x) \geq 0 \quad \text { on } M .
$$

Let $u \in C^{2}(M)$ be a positive solution of the differential inequality

$$
\Delta u+a(x) u \geq K(x) u^{\sigma}
$$

for some $\sigma>1$. Assume that, for any fixed $0<\delta<s$, there exists a function $\varphi \in C^{2}([\delta, s])$ with the following properties:

$$
\begin{gathered}
\varphi^{\prime \prime}(t)+(m-1) z(t) \varphi^{\prime}(t)+p(t) \varphi(t) \geq 0, \quad \text { on }[\delta, s], \\
\text { i) } \left.\varphi^{\prime}(s)=\frac{1}{\operatorname{vol}\left(\partial B_{s}(p)\right)} ; \quad \text { ii }\right) \varphi^{\prime}(t) \geq 0 \text { on }[\delta, s], \\
\varphi(s)=0, \\
\frac{-\varphi(\delta)}{\varphi^{\prime}(\delta)} \leq c
\end{gathered}
$$

for some constant $c>0$ independent of $s$ and $\delta$. Then, up to choosing $\delta$ sufficiently small, (and consequently $\varphi$ ), there exists a constant $c_{1}>0$, independent of $\delta$ and $s$ such that for any $R \in[\delta, s), \bar{u}(s)$ satisfies

$$
\bar{u}(s) \geq c_{1} \varphi^{\prime}(\delta) \delta^{m-1}-\int_{R}^{s} \varphi(t) \operatorname{vol}\left(\partial B_{t}\right) \bar{K}_{\sigma}(t) \bar{u}(t)^{\sigma} d t .
$$


Proof. We fix $0<\delta<s, \varphi$ and $R \in[\delta, s]$. We consider the function $\varphi(r(x))$ on $M$. Because of (1.21), (1.22), (1.25) and (1.26) ii) we have

$$
\Delta \varphi(r)(x) \geq-a(x) \varphi(r(x))
$$

in the weak sense on $\overline{B_{s}(p)} / B_{\delta}(p)$. The second Green's identity, (1.26) i), (1.27) together with the definition (1.19) of $\bar{u}$, give

$$
\begin{aligned}
\bar{u}(s)= & \int_{B_{s} / B_{R}} u \Delta \varphi-\varphi \Delta u \\
& +\int_{B_{R} / B_{\delta}} u \Delta \varphi-\varphi \Delta u+\int_{\partial B_{\delta}} u\langle\nabla \varphi, \nabla r\rangle-\varphi\langle\nabla u, \nabla r\rangle .
\end{aligned}
$$

Observe that (1.24), (1.30), (1.23) and positivity of $u$ yield

$$
\int_{B_{R} / B_{\delta}} u \Delta \varphi-\varphi \Delta u \geq 0
$$

Similarly,

$$
\int_{B_{s} / B_{R}} u \Delta \varphi-\varphi \Delta u \geq-\int_{B_{s} / B_{R}} \varphi(r) K(x) u^{\sigma} .
$$

Next, a straightforward application of Hölder' s inequality shows

$$
\int_{\partial B_{t}} K(x) u^{\sigma} \geq \bar{K}_{\sigma}(t) \bar{u}(t)^{\sigma} \operatorname{vol}\left(\partial B_{t}\right)
$$

where $\bar{K}_{\sigma}(t)$ has been defined in (1.20). Putting together (1.33), (1.34) and using the co-area formula, we finally get

$$
\int_{B_{s} / B_{R}} u \Delta \varphi-\varphi \Delta u \geq-\int_{R}^{s} \varphi(t) \bar{K}_{\sigma}(t) \bar{u}(t)^{\sigma} \operatorname{vol}\left(\partial B_{t}\right) d t .
$$

We now take care of the boundary term in (1.31). On the one hand,

$$
\int_{\partial B_{\delta}} u\langle\nabla \varphi, \nabla r\rangle-\varphi<\langle\nabla u, \nabla r\rangle=\varphi^{\prime}(\delta) \bar{u}(\delta) \operatorname{vol}\left(\partial B_{\delta}\right)-\varphi(\delta) \int_{B_{\delta}} \Delta u .
$$

On the other hand,

$$
\operatorname{vol}\left(\partial B_{\delta}\right) \asymp \delta^{m-1} ; \quad \operatorname{vol}\left(B_{\delta}\right) \asymp \delta^{m} \quad \text { as } \quad \delta \rightarrow 0^{+} .
$$

Using positivity of $u,(1.37)$ and (1.28) it is easy to see that, up to choosing $0<$ $\delta \leq \delta_{0}$ sufficiently small, there exists a constant $c_{1}$, independent of $\delta$ and $s$, such that

$$
\int_{\partial B_{\delta}} u\langle\nabla \varphi, \nabla r\rangle-\varphi\langle\nabla u, \nabla r\rangle \geq c_{1} \varphi^{\prime}(\delta) \delta^{m-1} .
$$

Estimate (1.29) now follows from (1.31), (1.32), (1.35) and (1.38).

Later on, we shall need to produce sub-(super-) solutions of the linear equation

$$
\Delta u+a(x) u=0
$$

on $(M, g)$. We shall achieve this task by constructing radial sub-(super-) solutions on a "model" manifold which appropriately compares with $M$. We recall that a model, in the sense of Greene and $\mathrm{Wu},[\mathrm{G}-\mathrm{W}]$, is a Riemannian manifold with a pole $p$ such that every linear isometry $\varphi: T_{p}(M) \rightarrow T_{p}(M)$ is realized as the differential of an isometry $\Phi: M \rightarrow M$. In particular, the smooth metric $d s^{2}$ of 
$M$ can be represented, in polar coordinates on $M /\{p\}=(0,+\infty) \times S^{m-1}$, in the form

$$
d s^{2}=d r^{2}+g(r)^{2} d \theta^{2}
$$

where $r$ is the distance from $p, d \theta^{2}$ the canonical metric on $S^{m-1}$ and $g$ is a smooth function on $[0,+\infty)$ satisfying

$$
g^{(2 k)}(0)=0, \quad g^{\prime}(0)=1, \quad k=0,1,2, \ldots .
$$

From now on, a model, as described above, will be denoted by a triple $(M, p, g)$.

Next, given the differential operator $L=\Delta+a(x)$ on $M$ and $\Omega \subset \subset M$ with smooth boundary, we shall indicate with $\lambda_{1}^{L}(\Omega)$ the first eigenvalue of the Dirichlet problem on $\Omega$. We set

$$
\lambda_{1}^{L}(M)=\inf _{\Omega \subset \subset M} \lambda_{1}^{L}(\Omega) .
$$

Lemma 1.3. Let $(M, p, g)$ be a model and let $a(r) \in C^{o}([0,+\infty))$ be nonnegative. Let $u=\alpha \circ r$ be a positive, radial $C^{2}-$ solution on $M$ of

$$
\Delta u+a(r) u=0,
$$

and let $v=\beta \circ r$ be a positive radial $C^{2}$-subsolution of (1.41) on $M / B_{R}(p)$, for some $R>0$, such that $\beta^{\prime}(R)>0$. Then

$$
\sup \left\{\frac{u(x)}{v(x)}: \quad x \in M / B_{R}(p)\right\}<+\infty .
$$

Proof. First of all we observe that existence of $u>0$ as a solution of (1.41) implies, by [FC-S], that the operator $L=\Delta+a(r)$ satisfies

$$
\lambda_{1}^{L}(M) \geq 0 \text {. }
$$

Next, (1.41) yields

$$
\left\{\begin{array}{l}
\alpha^{\prime \prime}+(m-1) \frac{g^{\prime}(r)}{g(r)} \alpha^{\prime}+a(r) \alpha=0, \quad \text { on } \quad[0,+\infty), \\
\alpha^{\prime}(0)=0,
\end{array}\right.
$$

so that, nonnegativity of $a(r)$, positivity of $\alpha$ and (1.44) imply $\alpha^{\prime}(r) \leq 0$. Having made this observation, we choose $\xi>0$ to satisfy

$$
\xi \beta^{\prime}(R)>\alpha^{\prime}(R) \quad \text { and } \quad \xi \beta(R)>\alpha(R) .
$$

Next, we fix $\bar{R}>R$ and let $\varphi$ be a positive radial solution of the Dirichlet eigenvalue problem for $L$ on $B_{\bar{R}+1}(p)$. Because of (1.39) and (1.43) $\varphi$ satisfies

$$
\left\{\begin{array}{lr}
\varphi^{\prime \prime}+(m-1) \frac{g^{\prime}(r)}{g(r)} \varphi^{\prime}+a(r) \varphi \leq 0, \\
\varphi^{\prime}(0)=0, & \varphi(\bar{R}+1)=0 .
\end{array}\right.
$$

In particular

$$
\varphi^{\prime}(t) \leq 0 \quad \text { on }[0, \bar{R}+1) .
$$

On the interval $[R, \bar{R}]$ we consider the function

$$
w(t)=\alpha(t)-\xi \beta(t) .
$$


Because of our choice of $\xi$ it follows that

$$
\text { i) } w^{\prime}(R)<0 ; \quad \text { ii) } w(R)<0 .
$$

On the other hand, $w$ satisfies

$$
\Delta w+a(r) w \leq 0 \quad \text { on } \overline{B_{\bar{R}}(p)} / B_{R}(p) .
$$

But, on $\overline{B_{\bar{R}}(p)} / B_{R}(p), \varphi$ is positive and solves

$$
\Delta \varphi+a(r) \varphi \leq 0 .
$$

By the generalized maximum principle (see $[\mathrm{P}-\mathrm{W}$, Theorem 10, page 73$]$ ), we have either

$$
w=c \varphi
$$

for some negative constant $c$, or $\frac{w}{\varphi}$ has its negative absolute minimum at $\bar{R}$ or $R$. However, in this latter case, we must have

$$
\left(\frac{w}{\varphi}\right)^{\prime}(R) \geq 0
$$

But, from (1.47) and (1.48),

$$
w^{\prime}(R) \varphi(R)-\varphi^{\prime}(R) w(R)<0
$$

contradicting (1.50). We therefore conclude $w(\bar{R})<0$. Since $\bar{R}>R$ was chosen arbitrarily we have proved

$$
u \leq \xi w \quad \text { on } M / B_{R}(p) ;
$$

this shows the validity of (1.42).

\section{The CASE $\operatorname{Riem}_{(M, g)} \leq 0$}

The aim of this section is to prove a slightly more general version of Theorem A, that is, Theorem 2.1 below, together with some related consequences.

A careful analysis of the role of the various assumptions shows that our main result, in many situations, is best possible. The requirement on $\operatorname{Riem}_{(M, g)}$ makes computations particularly simple; thus proofs and remarks are fully developed without obscuring the underlying reasoning. A thorough understanding of this paragraph allows us to skip details in section 3 , where the various arguments are based on the same principles.

To clarify notation, from now on $b(t)$ will denote a function, defined for $t$ sufficiently large, satisfying either (1.1) iii) and iv) or (1.1) iii) and $v$ ) of Section 1. In many cases we shall choose for $b(t)$ one of the functions

$$
b_{1}(t)=t^{-2} ; \quad b_{2}(t)=t^{-2}(\log t)^{-1} ; \quad b_{3}(t)=t^{-2}(\log t \log \log t)^{-1}
$$

and so on. Furthemore, $\bar{u}$ and $\bar{K}_{\sigma}$ shall denote respectively, the spherical mean of $u$ and the weighted spherical mean of $K$ as defined in (1.19), (1.20).

Theorem 2.1. Let $(M, g)$ satisfy

$$
\operatorname{Riem}_{(M, g)} \leq 0 \text { on } M,
$$


and let $a(x), K(x) \in C^{o}(M)$ be such that

$$
\begin{aligned}
& a(x) \leq \frac{A}{r(x)^{2}} \quad \text { on } M \text { for some } 0 \leq A \leq \frac{(m-2)^{2}}{4}, \\
& K(x) \geq 0 \text { on } M .
\end{aligned}
$$

Suppose that, for some constants $\sigma>1, n \in N, n \geq 1$,

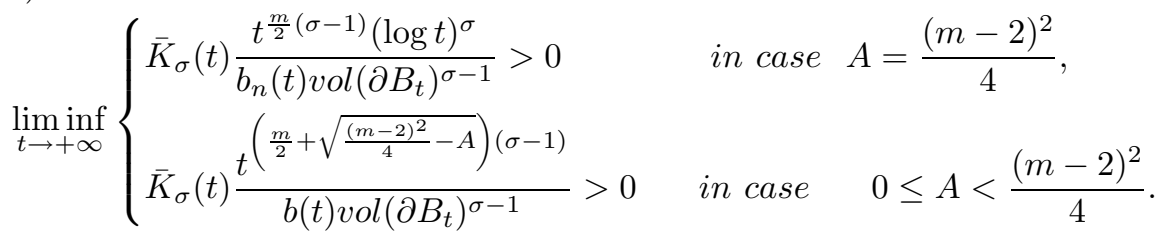

Then, the differential inequality

$$
\Delta u+a(x) u \geq K(x) u^{\sigma}
$$

has no positive $C^{2}$-solution on $M$.

Remark. We observe that the theorem is "a fortiori" true in case $A<0$. However, the result is sharp only for $0 \leq A \leq \frac{(m-2)^{2}}{4}$.

The same observation applies to the remaining results.

Proof. We reason by contradiction and assume that (2.5) has a positive $C^{2}$-solution $u$ on M. By the hessian comparision theorem and (2.1) we deduce

$$
\Delta r \geq \frac{m-1}{r}
$$

Next, we begin by considering the case $A=\frac{(m-2)^{2}}{4}$. We fix $0<\delta \leq R<s$ and define

$$
\varphi(t)=\frac{s^{\frac{m}{2}}}{\operatorname{vol}\left(\partial B_{s}\right)} t^{-\frac{m-2}{2}} \log \frac{t}{s} \quad \text { on } \quad[\delta, s] .
$$

Then $\varphi(t)$ satisfies (1.25) of Lemma 1.2 with $z(t)=\frac{1}{t},(1.26),(1.27),(1.28)$ up to choosing $0<\delta \leq \delta_{o}$ sufficiently small. From Lemma 1.2 it follows that $\bar{u}(s)$ satisfies (1.29). On the other hand,

$$
\varphi^{\prime}(\delta)=\frac{s^{\frac{m}{2}}}{\operatorname{vol}\left(\partial B_{s}\right)} \delta^{-\frac{m}{2}}\left\{1-\frac{m-2}{2} \log \frac{\delta}{s}\right\} .
$$

Therefore there exists a constant $c_{2}$, independent of $\delta$ and $s$, such that, in the notation of Lemma 1.2,

$$
c_{1} \varphi^{\prime}(\delta) \geq c_{2} \frac{s^{\frac{m}{2}} \log s}{\operatorname{vol}\left(\partial B_{s}\right)} .
$$

Furthermore, whenever $R \geq e$, say, and $s>R$, we have

$$
-\varphi(t)=\frac{s^{\frac{m}{2}} \log s}{\operatorname{vol}\left(\partial B_{s}\right)}\left(1-\frac{\log t}{\log s}\right) t^{-\frac{m-2}{2}} .
$$


From (2.8), (2.9) and (1.29) we therefore deduce that, on $[R,+\infty)$ with $R \geq e, \bar{u}(s)$ satisfies

$$
\bar{u}(s) \geq \frac{s^{\frac{m}{2}} \log s}{\operatorname{vol}\left(\partial B_{s}\right)}\left\{c_{2} \delta^{m-1}+\int_{R}^{s}\left(1-\frac{\log t}{\log s}\right) t^{-\frac{m-2}{2}} \bar{K}_{\sigma}(t) \operatorname{vol}\left(\partial B_{t}\right) \bar{u}(t)^{\sigma} d t\right\} .
$$

We let

$$
l(t)=t^{-\frac{m-2}{2}} \operatorname{vol}\left(\partial B_{t}\right) \bar{K}_{\sigma}(t) ; \quad \Psi(t)=\frac{t^{\frac{m}{2}} \log t}{\operatorname{vol}\left(\partial B_{t}\right)}
$$

and observe that, because of (2.4), all of the conditions of Lemma 1.1 and Remark 1.4 are satisfied. Contradiction.

The case $0 \leq A<\frac{(m-2)^{2}}{4}$ is treated similarly choosing the test function $\varphi$ as follows. Set

$$
a=-\frac{m-2}{2}+\sqrt{\frac{(m-2)^{2}}{4}-A}, \quad b=-\frac{m-2}{2}-\sqrt{\frac{(m-2)^{2}}{4}-A},
$$

and define

$$
\varphi(t)=\frac{1}{(a-b) \operatorname{vol}\left(\partial B_{s}\right)}\left[s^{1-a} t^{a}-s^{1-b} t^{b}\right] \quad \text { on } \quad[\delta, s] .
$$

We observe that

$$
c_{1} \varphi^{\prime}(\delta) \geq c_{2} \frac{s^{1-b}}{(a-b) \operatorname{vol}\left(\partial B_{s}\right)}
$$

and

$$
-\varphi(t)=\frac{s^{1-b}}{(a-b) \operatorname{vol}\left(\partial B_{s}\right)}\left[1-\left(\frac{t}{s}\right)^{a-b}\right] t^{b}
$$

for $0<t \leq s$ and some constant $c_{2}>0$, independent of $\delta$ and $s$.

Remark 2.14. The conclusion of Theorem 2.1 cannot be in general improved to assert the nonexistence of positive $C^{2}$-solutions of (2.5) outside a compact subset of $M$ even imposing reasonably stronger assumptions on $a(x)$ and $K(x)$. Somehow, this is due to the fact that the non-linearity in (2.5) is "sufficiently mild" (see, for instance [B]). Indeed, consider euclidean space $R^{m}$ with $m \geq 2$. For any fixed positive constants $R, c, b, \sigma$, let

$$
a(x)=-c \quad \text { and } \quad K(x)=e^{b|x|} \quad \text { on } \quad R^{m} .
$$

Define

$$
u(x)=e^{\alpha b|x|}
$$

for some $\alpha \in R$. Then, up to choosing $\alpha$ sufficiently negative, $u$ satisfies (2.5) on $R^{m} / B_{R}(0)$.

Next, we shall state a geometric consequence of Theorem 2.1. We recall that given a manifold $(M, g)$ with a pole $p$, the radial curvatures of $M$ at $q$ are the sectional curvatures evaluated on 2-planes containing the tangent vector of the geodesic ray connecting $p$ to $q$.

In order to avoid a condition on $K(x)$ involving $\operatorname{Vol}\left(\partial B_{r(x)}\right)$ we estimate this latter from above with the aid of the following result whose proof is given in [RRS2]. 
Proposition 2.2. Let $(M, g)$ satisfy

$$
\operatorname{Ricc}_{(M, g)}(\nabla r, \nabla r) \geq-(m-1) B^{2}\left(1+r^{2}\right)^{\frac{\alpha}{2}}
$$

for some constants $B>0, \alpha \geq-2$. Then, the following inequality holds weakly:

$$
\Delta r \leq(m-1) H r^{\frac{\alpha}{2}} \quad \text { for } r \gg 1,
$$

with

$$
H= \begin{cases}B \quad & \text { if } \quad \alpha \geq 0, \\ \frac{1}{4}\left(-\alpha+\sqrt{\alpha^{2}+16 B^{2}}\right) & \text { if }-2 \leq \alpha<0 .\end{cases}
$$

As a consequence of Proposition 2.2, in the assumption (2.14), we obtain

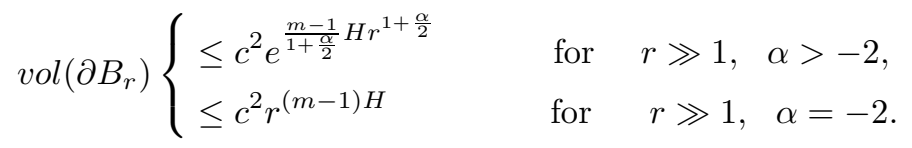

Corollary 2.3. Let $(M, g)$ be a manifold with a pole $p$, nonpositive radial curvature and scalar curvature $s(x)$ satisfying

$$
s(x) \geq-(m-2)(m-1) \frac{1}{r(x)^{2}} .
$$

Assume that the Ricci tensor in the radial direction satisfies (2.14). Given $K(x) \in$ $C^{\infty}(M)$ suppose that

$$
K(x) \leq \begin{cases}-c^{2} \frac{e^{\left.4 \frac{m-1}{(m-2)\left(1+\frac{\alpha}{2}\right.}\right)} H r^{1+\frac{\alpha}{2}}}{r(x)^{\frac{2 m}{m-2}+2}[\log r(x)]^{\frac{2 m}{m-2}}[\log \log r(x)]} & \text { if } \quad \alpha>-2, \\ -c^{2} \frac{r^{2 \frac{m-1}{m-2}\left(1+\sqrt{1+4 B^{2}}\right)}}{r(x)^{\frac{2 m}{m-2}+2}[\log r(x)]^{\frac{2 m}{m-2}}[\log \log r(x)]} & \text { if } \quad \alpha=-2\end{cases}
$$

for $r(x) \gg 1$ and some constant $c>0$. Then, if

$$
K(x) \leq 0 \quad \text { on } M
$$

the metric $g$ cannot be conformally deformed to a metric of scalar curvature $K(x)$.

Proof. First of all we note that (2.6) holds under the present assumptions. Furthermore if $g_{u}=u^{\frac{4}{m-2}} g, u>0$, is a conformal deformation of the metric $g$ with scalar curvature $K(x)$, then $u$ satisfies

$$
c_{m} \Delta u-s(x) u+K(x) u^{\frac{m+2}{m-2}}=0 \quad \text { on } \quad M
$$

with $c_{m}=\frac{4(m-1)}{m-2}$. The result now follows from Theorem 2.1.

Remark 2.21. A further result along these lines is given in Corollary 4.2 of [RRS1], under assumptions on the Ricci curvature alone.

As already observed in the Introduction, Theorem 2.1 complements recent results of [C-L] section 2, obtained when $M=R^{m}$ and $a(x) \equiv 0$. For the sake of clarity and ease of comparison we explicitly state 
Corollary 2.4. Let $a(x), K(x) \in C^{o}\left(R^{m}\right), m \geq 3$, satisfy (2.2) and (2.3) on $R^{m}$. Suppose that, for some constant $\sigma>1$ and $n \in N, n \geq 1$,

i) $\liminf _{t \rightarrow+\infty}\left\{\begin{array}{l}\bar{K}_{\sigma}(t) \frac{t^{-\frac{m-2}{2}(\sigma-1)}(\log t)^{\sigma}}{b_{n}(t)}>0 \quad \text { in case } A=\frac{(m-2)^{2}}{4}, \\ \bar{K}_{\sigma}(t) \frac{t^{\left(-\frac{m-2}{2}+\sqrt{\frac{(m-2)^{2}}{4}-A}\right)(\sigma-1)}}{b(t)}>0 \quad \text { in case } 0 \leq A<\frac{(m-2)^{2}}{4}\end{array}\right.$

with $b(t)$ defined for $t$ sufficiently large and satisfying

j)

$$
t b(t) \notin L^{1}(+\infty) ;
$$

k) there exist $R_{1}, \eta \in(0,1]$ such that

$$
\int_{R_{1}}^{t} s b(s) d s \quad \text { is strictly increasing; } \quad \liminf _{t \rightarrow+\infty} \int_{R_{1}}^{t} s b(s) d s-\eta t^{2} b(t)>-\infty .
$$

Then the differential inequality

l)

$$
\Delta u+a(x) u \geq K(x) u^{\sigma}
$$

has no positive $C^{2}$-solution on $R^{m}$.

Next, we discuss the assumptions of Theorem 2.1. We begin with

$$
a(x) \leq \frac{A}{r(x)^{2}} \quad \text { on } \quad M \quad \text { with } \quad 0 \leq A \leq \frac{(m-2)^{2}}{4} .
$$

In order to motivate this request we make the following elementary observation. We want to allow, as in $(2.3), K(x)$ to be small on a compact set $\bar{D} \subset M$. On $D$, (2.5) becomes

$$
\Delta u+a(x) u \geq 0
$$

It is then clear that the nonexistence of a positive $C^{2}$-solution $u$ on $M$ of (2.5) might be due to the fact that solutions of the equation

$$
\Delta u+a(x) u=0 \quad \text { on } \quad D
$$

have to have zeros in $\mathrm{D}$. This could conceivably be the case when $a(x)$ is positive and sufficiently large. On the other hand, condition (2.2) implies that a nonnegative solution $u$ on $M$ of $(2.21)$, with $u(p)>0$ for some $p \in M$, has to have a positive mean $\bar{u}$ on $[0,+\infty)$. Therefore, an assumption like $(2.2)$ is certainly what we expect to require, in order to have $u$ positive, in case $\Delta$ and $a(x)$ are "radial". We state this result carefully. Its proof is contained in that of Theorem 2.1 .

Theorem 2.5. Let $(M, g)$ satisfy

$$
\operatorname{Riem}_{(M, g)} \leq 0 \text { on } M,
$$

and let $a(x) \in C^{o}(M)$ be such that (2.2) holds true. Let $u$ be a nonnegative $C^{2}-$ solution of $(2.21)$ on $B_{r}(p)$ with $u(p)>0$. Then

$$
\bar{u}(r) \geq \begin{cases}c \frac{r^{\frac{m}{2}} \max \{\log r, 1\}}{\operatorname{vol}\left(\partial B_{r}\right)} & \text { in case } A=\frac{(m-2)^{2}}{4}, \\ c \frac{r^{\frac{m}{2}+\sqrt{\frac{(m-2)^{2}}{4}}-A}}{\operatorname{vol}\left(\partial B_{r}\right)} & \text { in case } 0 \leq A<\frac{(m-2)^{2}}{4}\end{cases}
$$

for some constant $c>0$ independent of $r$. 
Remark 2.25. Of course, Theorem 2.5 can be restated for a manifold with a pole relaxing the requirement on the sectional curvature to the corresponding one on the radial curvature.

The above observation has shifted our original discussion about (2.2) onto the analysis of Theorem 2.5. In this regard we first observe that the decay of the RHS of (2.2) at infinity cannot be relaxed however small, but otherwise positive, we take $A$. Indeed, we consider the case $(M, g)=R^{m}$, with its canonical flat metric. Let

$$
a(x)=\tilde{a}(|x|)=\tilde{a}(r(x))
$$

with $\tilde{a}(r) \in C^{o}([0,+\infty))$ satisfying

$$
\tilde{a}(r) \begin{cases}\leq \frac{A}{r^{2}}, & r \leq R_{o}, \\ =\frac{A \phi(r)}{r^{2}}, & r>R_{o},\end{cases}
$$

for some $\phi(r) \rightarrow+\infty$ as $r \rightarrow+\infty, \phi$ nondecreasing, $A>0$ and $R_{o}>0$. Suppose that $u$ is a positive $C^{2}$-solution of

$$
\Delta u+a(x) u=0
$$

on $R^{m}$. Then, as is easily seen, $\bar{u}$ satisfies

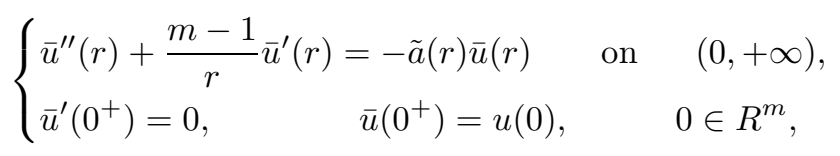

with $\bar{u}>0$ on $[0,+\infty)$. Let $t=(m-2) r^{m-2}$ and set

$$
\gamma(t)=t \bar{u}\left(\left[\frac{t}{m-2}\right]^{\frac{1}{m-2}}\right) \quad \text { on } \quad(0,+\infty) .
$$

A simple computation shows that $\gamma(t)$ satisfies

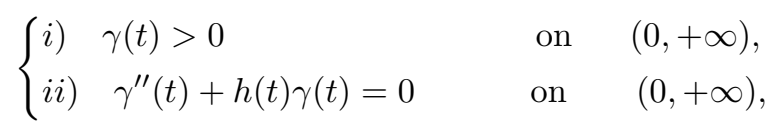

with

$$
h(t)=\frac{A}{(m-2)^{2}} \frac{1}{t^{2}} \phi\left(\left[\frac{t}{m-2}\right]^{\frac{1}{m-2}}\right) \quad \text { for } \quad t \gg 1 .
$$

On the other hand, since $\phi$ is nondecreasing and diverges at $+\infty$, we have

$$
\liminf _{t \rightarrow+\infty} t \int_{t}^{+\infty} h(s) d s \geq \liminf _{t \rightarrow+\infty} \frac{A}{(m-2)^{2}} \phi\left(\left[\frac{t}{m-2}\right]^{\frac{1}{m-2}}\right)=+\infty .
$$

Hence, Hille's criterion implies that (2.28) ii) is oscillatory, that is, (2.26) cannot have positive $C^{2}$-solutions on $R^{m}$.

We note that

$$
\liminf _{t \rightarrow+\infty} \frac{A}{(m-2)^{2}} t \int_{t}^{+\infty} \frac{d s}{s^{2}}\left\{\begin{array}{lll}
>\frac{1}{4} & \text { if } & A>\frac{(m-2)^{2}}{4}, \\
=\frac{1}{4} & \text { if } & A=\frac{(m-2)^{2}}{4} .
\end{array}\right.
$$


Thus (2.28) ii), with $\phi \equiv 1$, is oscillatory whenever $A>\frac{(m-2)^{2}}{4}$; while for $A=$ $\frac{(m-2)^{2}}{4}$ we are in the dubious case of Hille's criterion. This shows that the range of $A$ in (2.2) is sharp.

The next example sheds light on the role of $(2.2)$ on compact subsets of $(M, g)$. Again we consider the case of Euclidean space $R^{m}$. Let $b>0$ be a constant and define $a(r)$ on $[0,+\infty)$ to be continuous, positive and such that

$$
a(r)\left\{\begin{array}{lll}
=b^{2}(m-2)^{2} r^{2(m-3)} & \text { on } & {\left[0,\left(\frac{\pi}{b}\right)^{\frac{1}{m-2}}\right],} \\
\leq \frac{(m-2)^{2}}{4} \frac{1}{r(x)^{2}} & \text { on } \quad\left[\left(\frac{\pi}{b}\right)^{\frac{1}{m-2}}+1,+\infty\right) .
\end{array}\right.
$$

Then, the problem

$$
\left\{\begin{array}{l}
\varphi^{\prime \prime}(r)+\frac{m-1}{r} \varphi^{\prime}(r)+a(r) \varphi(r)=0, \\
\varphi(0)=b, \quad \varphi^{\prime}(0)=0
\end{array}\right.
$$

has a unique solution $\varphi$ on $[0,+\infty)$. However, because of $(2.31)$, it is immediate to see that

$$
\varphi(r)=\frac{\sin \left(b r^{m-2}\right)}{r^{m-2}} \quad \text { on } \quad\left(0,\left(\frac{\pi}{b}\right)^{\frac{1}{m-2}}\right] .
$$

We observe that $u(x)=\varphi(|x|)$ is a $C^{2}$-solution on $R^{m}$ of $\Delta u+a(r(x)) u=0$ with $u(0)=b>0$. But $\varphi$ has a zero in $r_{o}=\left(\frac{\pi}{b}\right)^{\frac{1}{m-2}}$ so that $u \equiv 0$ on $\partial B_{(\pi / b)^{\frac{1}{m-2}}}(0)$, $0 \in R^{m}$.

It remains to show that conclusion (2.24) of Theorem 2.6 is sharp. This fact is contained in Proposition 4.1 below (see Remark 4.13).

We return to Theorem 2.1. As for assumption (2.4) we show its sharpness by means of the following counterexample. Let

$$
\begin{aligned}
& \tilde{a}(t)=\frac{(m-2)^{2}}{4} \frac{1}{(t+e)^{2}} ; \\
& \alpha(t)=(t+e)^{-\frac{m-2}{2}}\left\{-\frac{m-4}{m-2}+\frac{2 \epsilon}{(\sigma-1)(m-2)}+[\log (t+e)]^{1+\frac{\epsilon}{\sigma-1}}\right\}
\end{aligned}
$$

for some $\epsilon>0, \sigma>1, m \geq 3$. We note that $\alpha(t)>0$ on $[0,+\infty)$ and $\alpha^{\prime}(0)=0$.

It is not hard to verify that we can choose $\epsilon$ sufficiently large in such a way that there exists $\tilde{K}(t)$, positive and continuous on $[0,+\infty)$, with

$$
\tilde{K}(t) \sim c \frac{t^{\frac{m-2}{2}(\sigma-1)-2}}{(\log t)^{\sigma+1+\epsilon}} \quad \text { as } \quad t \rightarrow+\infty,
$$

for some constant $c>0$, for which $\alpha$ satisfies

$$
\left\{\begin{array}{l}
\alpha^{\prime \prime}+\frac{m-1}{t} \alpha^{\prime}+\tilde{a}(t) \alpha=\tilde{K}(t) \alpha^{\sigma} \text { on } \quad[0,+\infty), \\
\alpha^{\prime}(0)=0, \quad \alpha(0)>0 .
\end{array}\right.
$$

On $R^{m}$ we define $a(x)=\tilde{a}(|x|), K(x)=\tilde{K}(|x|), u(x)=\alpha(|x|)$. Then $u$ is a positive (radial) $C^{2}$-solution of (0.1) on $R^{m}$. On the other hand, all of the assumptions of Theorem 2.1 are satisfied except for condition (2.4), with $A=\frac{(m-2)^{2}}{4}$, which barely fails to be met. 
In case $0 \leq A<\frac{(m-2)^{2}}{4}$ we let

$$
\begin{aligned}
& \tilde{a}(t)=\frac{A}{(t+e)^{2}}, \quad \beta=\sqrt{\frac{(m-2)^{2}}{4}-A}, \\
& \alpha(t)=(t+e)^{-\frac{m-2}{2}+\beta}\left\{-1+\frac{2 \epsilon}{(\sigma-1)(m-2-2 \beta)}+[\log (t+e)]^{\frac{\epsilon}{\sigma-1}}\right\},
\end{aligned}
$$

for some $\epsilon>0, \sigma>1, m \geq 3$. Up to choosing $\epsilon$ sufficiently large, we see that $\alpha$ satisfies (2.34) where now the asymptotic behavior of $\tilde{K}(t)$ is

$$
\tilde{K}(t) \sim c \frac{t^{\left(\frac{m-2}{2}-\sqrt{\frac{(m-2)^{2}}{4}-A}\right)(\sigma-1)-2}}{(\log t)^{1+\epsilon}} \quad \text { as } t \rightarrow+\infty,
$$

for some constant $c>0$. The rest is as above.

As for the assumptions on the function $b(t)$ we note that (1.1) iii) is essential. Indeed, Naito [Na] shows that if $|K(x)| \leq b(|x|)$ on $R^{m}$ for some $b(t)$ such that $t b(t) \in L^{1}(+\infty)$, then the equation

$$
\Delta u=K(x) u^{\sigma}, \quad \sigma>1,
$$

has infinitely many bounded positive solutions on $R^{m}$ which tend to a positive constant at $\infty$.

\section{THE CASE $\operatorname{Riem}_{(M, g)} \leq-B^{2}$}

A careful analysis of the proof of Theorem 2.1 clarifies the role of assumptions (2.1). As matter of fact, it is possible to give versions of this theorem under a different curvature request. For instance, we may assume

$$
\operatorname{Riem}_{(M, g)} \leq-B^{2}\left(1+r^{2}\right)^{\frac{\alpha}{2}}
$$

for some constants $B>0$ and $\alpha \geq-2$. Indeed, once an appropriate choice of the test function $\varphi$ in (2.7) has been made, the argument of Theorem 2.1 goes through without changes. However, a general assumption like (3.1) makes computational details quite cumbersome without adding any further insight. Therefore, to avoid unpleasant complications and motivated by geometrical reasons, we limit ourselves to the case $\alpha=0$.

Theorem 3.1. Let $(M, g)$ be such that $m \geq 2$ and

$$
\operatorname{Riem}_{(M, g)} \leq-B^{2}
$$

for some constant $B>0$. Let $a(x), K(x) \in C^{o}(M)$ satisfy

$$
\begin{gathered}
a(x) \leq A B^{2} \operatorname{coth}[B r(x)] \quad \text { on } M \text { with } 0 \leq A \leq \frac{(m-1)^{2}}{4}, \\
K(x) \geq 0 \text { on } M .
\end{gathered}
$$

Suppose that, for some constant $\sigma>1$,

$$
\liminf _{t \rightarrow+\infty}\left\{\begin{array}{l}
\bar{K}_{\sigma}(t) \frac{t^{\sigma-1} e^{\frac{m-1}{2} B(\sigma-1) t}}{b(t) \operatorname{vol}\left(\partial B_{t}\right)^{\sigma-1}}>0 \\
\bar{K}_{\sigma}(t) \frac{e^{B\left(\frac{m-1}{2}+\sqrt{\frac{(m-1)^{2}}{4}-A}\right)(\sigma-1) t}}{t b(t) \operatorname{vol}\left(\partial B_{t}\right)^{\sigma-1}}>0 \quad \text { in case } A=\frac{(m-1)^{2}}{4},
\end{array}\right.
$$


with $b(t)$ satisfying (1.1) iii), $v)$. Then, the differential inequality

$$
\Delta u+a(x) u \geq K(x) u^{\sigma}
$$

has no positive $C^{2}$-solution on $M$.

Proof. First of all we observe that, by the hessian comparision theorem,

$$
\Delta r \geq(m-1) B \operatorname{coth} B r \quad \text { on } \quad M \text {. }
$$

From now on the proof follows the argument of Theorem 2.1. In case $A=\frac{(m-1)^{2}}{4}$ we choose as test function on $[\delta, s]$,

$$
\varphi(t)=\frac{t-s}{\operatorname{vol}\left(\partial B_{s}\right)} e^{\frac{m-1}{2} B(s-t)}
$$

while in case $0 \leq A<\frac{(m-1)^{2}}{4}$ we choose

$$
\varphi(t)=-\frac{e^{a B(s-t)}\left[1-e^{-a B(s-t)}\right]}{a \operatorname{Bvol}\left(\partial B_{s}\right)}
$$

with

$$
a=\frac{m-1}{2}+\sqrt{\frac{(m-1)^{2}}{4}-A} .
$$

Corollary B of the Introduction is an immediate consequence of Theorem 3.1 (see also Corollary 2.3). We observe that assumption (0.6) can be substituted with

$$
K(x)<0 \quad \text { for } r(x) \gg 1
$$

and

$$
\liminf _{t \rightarrow+\infty} \overline{(-K)_{\sigma}}(t) \frac{e^{\frac{2 m}{m-2} B t}}{t b(t) \operatorname{vol}\left(\partial B_{t}\right)^{\frac{4}{m-2}}}>0 .
$$

In particular in case $(M, g)$ is hyperbolic space $H^{m}$ with its standard metric of constant sectional curvature -1 , the choice

$$
b(t)=t^{-2}(\log t \log \log t \ldots)^{-1}
$$

in $(3.10)$ gives

$$
\liminf _{t \rightarrow+\infty} \overline{(-K)}_{\sigma}(t) \frac{t \log t \log \log t \ldots}{e^{2 t}}>0 .
$$

From [RRV2] we know that in this case (3.11) is "best possible".

From the proof of Theorem 3.1 we derive a result analogous to Theorem 2.5. We shall make use of it in section 4 .

Theorem 3.2. Let $(M, g)$ satisfy $m \geq 2$ and (3.2) and let $a(x) \in C^{o}(M)$ be such that (3.3) holds true. Let $u$ be a nonnegative $C^{2}$-solution of

$$
\Delta u+a(x) u \geq 0
$$

on $B_{r}(p)$, with $u(p)>0$. Then,

$$
\bar{u}(r) \geq c \frac{r e^{\frac{m-1}{2} B r}}{\operatorname{vol}\left(\partial B_{r}\right)}
$$

for some constant $c>0$ independent of $r$. 
Analogously to what has been done for Theorem 2.5 it is possible to construct examples which show that assumption (3.3) and conclusion (3.12) are "best possible". We leave the details to the interested reader (see also Proposition 4.1 and Remark 4.13 below).

\section{A uniqueness Result}

The aim of this section is to provide a proof of Theorem C. Towards this end we need a preliminary result which may be of independent interest.

Proposition 4.1. Let $(M, g)$ have dimension $m$. Suppose that

$$
\operatorname{Riem}_{(M, g)} \leq-B^{2}
$$

for some constant $B \geq 0$. Depending on whether $B>0$ or $B=0$, assume respectively that $m \geq 2$ or $m \geq 3$. Let $a(x) \in C^{o}(M)$ satisfy

$$
\begin{cases}\text { i) } a(x) \leq \frac{(m-1)^{2}}{4} B^{2} \operatorname{coth}[B r(x)] & \text { if } B>0, \\ \text { ii) } a(x) \leq \frac{(m-2)^{2}}{4} \frac{1}{r(x)^{2}} & \text { if } B=0 .\end{cases}
$$

Then, there exists a positive $C^{2}$-supersolution $w$ of

$$
\Delta w+a(x) w=0
$$

such that

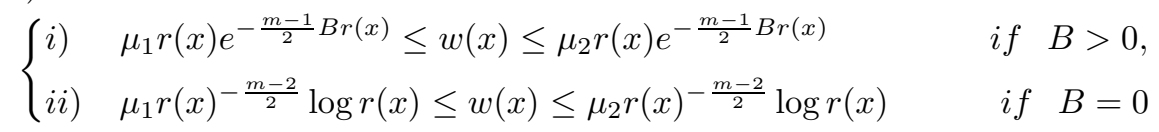

for some positive constants $\mu_{1}, \mu_{2}$, and $r(x)$ sufficiently large.

Proof. We deal with the case $B>0$, the remaining one being completely similar (see also Remark 4.13 below).

We choose $\hat{a}(r) \in C^{o}([0,+\infty))$ so as to satisfy

$$
\text { i) } \hat{a}(r)>0 \quad \text { on } \quad[0,+\infty) ; \quad \text { ii }) \quad a(x) \leq \hat{a}(r(x)) \quad \text { on } \quad M \text {, }
$$

and, for some fixed $\epsilon>0$,

$$
\hat{a}(r) \begin{cases}=\frac{(m-1)^{2}}{4} B^{2} \operatorname{coth} B r & \text { on } \quad[\epsilon,+\infty), \\ \leq \frac{(m-1)^{2}}{4} B^{2} \operatorname{coth} B r & \text { on } \quad[0, \epsilon) .\end{cases}
$$

Next, we consider hyperbolic space $\left(H^{m}, d s^{2}\right)$ with constant sectional curvature $-B^{2}$. On $H^{m} /\{0\}=(0,+\infty) \times S^{m-1}$ we represent the metric in the form

$$
d s^{2}=d t^{2}+\sinh ^{2}(B t) d \theta^{2}
$$

with $d \theta^{2}$ the canonical metric on $S^{m-1}$ and $t(x)=\operatorname{dist}_{\left(H^{m}, d s^{2}\right)}(x, 0)$. On $H^{m}$ the equation

$$
\Delta_{d s^{2}} u+\hat{a}(r) u=0
$$


has a positive radial $C^{2}$-solution. Indeed, by (4.7), a radial solution of (4.8) is of the form $u=\alpha \circ t$ with $\alpha$ a solution of

$$
\left\{\begin{array}{l}
\alpha^{\prime \prime}(t)+(m-1) B \operatorname{coth}(B t) \alpha^{\prime}(t)+\hat{a}(t) \alpha(t)=0 \quad \text { on } \quad[0,+\infty), \\
\alpha^{\prime}(0)=0, \quad \alpha(0)=\alpha_{0}
\end{array}\right.
$$

It is well known that $\alpha$ can be obtained via the Picard iteration procedure, and, having chosen $\alpha_{0}>0$, so that $u(0)>0$, Theorem 3.2 immediately implies that $u$ is positive on $H^{m}$. We fix $b>0$ and consider

$$
v(t(x))=[t(x)-b][\sinh B t(x)]^{-\frac{m-1}{2}} \quad \text { on } \quad(b,+\infty) .
$$

It is immediate to verify that $v$ is a positive radial subsolution of (4.8) on $H^{m} / \overline{B_{b}(0)}$. Furthermore,

$$
v^{\prime}(b+\epsilon)>0
$$

for some $\epsilon>0$ sufficiently small. Then, according to Lemma 1.3 and Theorem 3.2, $u(x)=\alpha(t(x))$ satisfies

$$
\mu_{1} t(x) e^{-\frac{m-1}{2} B t(x)} \leq u(x) \leq \mu_{2} t(x) e^{-\frac{m-1}{2} B t(x)}
$$

for some positive constants $\mu_{1}, \mu_{2}$ and $t$ sufficiently large. We define on $(M, g)$

$$
w(x)=\alpha(r(x)) .
$$

It is then clear that $w$ satisfies (4.4) $i$ ). Furthemore, from (4.5) $i$ ) and (4.9) we have

$$
\alpha^{\prime}(t) \leq 0 \quad \text { on } \quad[0,+\infty) .
$$

On the other hand, by the hessian comparision theorem, (3.7) holds on $M$. Putting together (4.11), (4.9), (3.7) and (4.5) ii) it is immediate to see that $w$ is a positive $C^{2}$-supersolution of (4.3).

Remark 4.13. The proof of Proposition 4.1 shows that, in the assumptions of Theorem 3.2, conclusion (3.12) is sharp. Indeed, the solution $u$ of $(4.8)$ on $\left(H^{m}, d s^{2}\right)$ satisfies (4.10). The same observation applies in case $B=0$. It is enough to choose $\hat{a}(r)$ to satisfy (4.5) and

$$
\hat{a}(r)\left\{\begin{array}{lll}
=\frac{(m-2)^{2}}{4} \frac{1}{r(x)^{2}} & \text { on } & {[1+\epsilon,+\infty),} \\
\leq \frac{(m-2)^{2}}{4} \frac{1}{r(x)^{2}} & \text { on } & {[0,1+\epsilon)}
\end{array}\right.
$$

for some $\epsilon>0$; then we work on $R^{m}$ instead of $\left(H^{m}, d s^{2}\right)$ and we choose

$$
v(t(x))=t(x)^{-\frac{m-2}{2}} \log t(x) \quad \text { on } \quad R^{m} / B_{1+\epsilon}(0) .
$$

We note that $v^{\prime}(1+\epsilon)>0$ for $\epsilon$ sufficiently small. In particular, conclusion (2.24) of Theorem 2.5 is sharp (at least when $A=\frac{(m-2)^{2}}{4}$; but the general case can be dealt with similarly).

The proof of Theorem C now follows from Proposition 4.1 and Theorem 3.1 of [RRV1]. However, for the sake of completeness and since the present is a special case, we report the argument here. 
Proof (of Theorem C). We let $w$ be as in Proposition 4.1 and we fix $\epsilon>0$. Given $u$ and $v$ positive solutions of (0.1) we define

$$
\varphi=\varphi_{\epsilon}=u+\epsilon w>0 \quad \text { on } \quad M .
$$

It is a simple matter to check that $(0.1),(0.9) i)$, together with the fact that $w$ is a supersolution of (4.3), the nonnegativity of $u$, and the elementary inequality

$$
(u+\epsilon w)^{\sigma}-u^{\sigma} \geq \epsilon \sigma u^{\sigma-1} w
$$

imply that

$$
\Delta \varphi+a(x) \varphi-K(x) \varphi^{\sigma} \leq 0 \quad \text { on } \quad M .
$$

Thus (4.17) and (0.1) finally give

$$
\frac{\Delta v}{v}-\frac{\Delta \varphi}{\varphi}-K(x)\left[v^{\sigma-1}-\varphi^{\sigma-1}\right] \geq 0 .
$$

Next, we observe that (0.1), (4.4) i) (or ii) ), $u, v, w>0$, imply that

$$
\left(v^{2}-\varphi^{2}\right)^{+} \in C^{o}(M) \cap H_{2}^{1}(M)
$$

is compactly supported. On the other hand, for any $\gamma \in C_{o}^{\infty}(M)$, we have

$$
\operatorname{div}\left[\gamma\left(\frac{\nabla v}{v}-\frac{\nabla \varphi}{\varphi}\right)\right]=\gamma \operatorname{div}\left(\frac{\nabla v}{v}-\frac{\nabla \varphi}{\varphi}\right)+\left\langle\nabla \gamma, \frac{\nabla v}{v}-\frac{\nabla \varphi}{\varphi}\right\rangle .
$$

Thus, having set

$$
\Theta=\{x \in M: v(x)>\varphi(x)\},
$$

it is easy to deduce from (4.19) and the divergence theorem that

$$
\int_{\Theta}\left(v^{2}-\varphi^{2}\right)^{+}\left(\frac{\Delta v}{v}-\frac{\Delta \varphi}{\varphi}\right)+\left|\nabla v-\frac{v}{\varphi} \nabla \varphi\right|^{2}+\left|\nabla \varphi-\frac{\varphi}{v} \nabla v\right|^{2}=0 .
$$

Therefore, (4.18) and (4.20) imply that

$$
\int_{\Theta} K(x)\left(v^{2}-\varphi^{2}\right)^{+}\left[v^{\sigma-1}-\varphi^{\sigma-1}\right]+\left|\nabla v-\frac{v}{\varphi} \nabla \varphi\right|^{2}+\left|\nabla \varphi-\frac{\varphi}{v} \nabla v\right|^{2} \leq 0 .
$$

Since $K(x) \geq 0$, it follows that $\frac{v}{\varphi}$ is constant on any connected component of $\Theta$, if not empty. Let $\Omega$ be a connected component in the compact set $\Theta$; then $\frac{v}{\varphi}=c(\Omega)$ on $\Omega$. As $\frac{v}{\varphi}(x)$ tends to 1 as $x$ approaches $\partial \Omega$, we deduce that $c(\Omega)=1$ and thus $\Omega=\emptyset$. Hence $\Theta=\emptyset$ and

$$
v \leq \varphi=u+\epsilon w
$$

for any $\epsilon>0$; that is, $v \leq u$ on $M$. Reversing the role of $u$ and $v$ we deduce $u \equiv v$ on $M$.

In Proposition 4.1 we have guaranteed positivity of the solution $\alpha$ of (4.9), with $\alpha_{o}>0$, via Theorem 3.2. As a matter of fact, we can use this latter result and its relative, Theorem 2.5, to study positivity of solutions of a problem of the type

$$
\left\{\begin{array}{l}
\alpha^{\prime \prime}(t)+h(t) \alpha^{\prime}(t)+a(t) \alpha(t)=0 \quad \text { on } \quad[0,+\infty), \\
\alpha(0)>0, \quad \alpha^{\prime}(0)=0
\end{array}\right.
$$

for some given $h(t)$ and $a(t)$.

To illustrate this technique we focus our attention on Theorem 2.5. Similar results can be obtained with the aid of Theorem 3.2 or a combination of both. 
Proposition 4.2. Let $m \geq 3$ and $\xi(t) \in C^{\infty}([0,+\infty))$ be such that

$$
\begin{gathered}
\xi^{(2 n)}(0)=0, \quad n=0,1,2, \ldots, \\
(m-1) t \xi^{\prime}(t)+t \xi(t)^{2}+2(m-1) \xi(t) \geq 0 \quad \text { on }[0,+\infty) .
\end{gathered}
$$

Let $a(t) \in C^{o}([0,+\infty))$ satisfy

$$
a(t) \leq \frac{(m-2)^{2}}{4} \frac{1}{t^{2}} \quad \text { on }[0,+\infty) .
$$

Then, any $C^{2}-$ solution of the initial value problem (4.22) with

$$
h(t)=\frac{m-1}{t}+\xi(t)
$$

is positive on $[0,+\infty)$.

Remark. Observe that any local solution of (4.22) is indeed defined on $[0,+\infty)$.

Proof. We define

$$
g(r)=e^{\frac{1}{m-1}\left\{\int_{1}^{r}\left[\frac{m-1}{s}+\xi(s)\right] d s+\int_{0}^{1} \xi(s) d s\right\}}
$$

and observe that $g$ satisfies

$$
g^{\prime \prime}(r)=-w(r) g(r), \quad \text { on } \quad[0,+\infty)
$$

with

$$
w(r)=-\left[\frac{1}{m-1} \xi^{\prime}(r)+\frac{1}{(m-1)^{2}} \xi(r)^{2}+\frac{2}{m-1} \frac{1}{r} \xi(r)\right] .
$$

Because of (4.23) and $\xi(r) \in C^{\infty}([0,+\infty))$, we have $w(r) \in C^{\infty}([0,+\infty))$ and $w^{(2 n+1)}(0)=0$ for each $n=0,1,2,3, \ldots$ Furthermore (4.24) gives

$$
w(r) \leq 0 .
$$

On $R^{m} /\{0\}=(0,+\infty) \times S^{m-1}$ we define the metric

$$
d s^{2}=d r^{2}+g(r)^{2} d \theta^{2}
$$

with $d \theta^{2}$ the canonical metric on $S^{m-1}$. (4.27), (4.28), (4.30) and a result of Kazdan and Warner $[\mathrm{K}-\mathrm{W}]$ imply that $d s^{2}$ has a smooth extension to a complete metric on $R^{m}$ with nonpositive radial curvature.

On the other hand (4.22) and (4.27) allow us to reinterpret $\alpha(t)$, the solution of $(4.22)$ on $[0,+\infty)$, as a radial $C^{2}$-solution, $u=\alpha \circ r$, on the model $\left(R^{m}, 0, d s^{2}\right)$, of the equation

$$
\Delta_{d s^{2}} u+a(r) u=0 .
$$

Note that $u(0)=\alpha(0)>0$. Let $B_{R}(0)$ be the maximal geodesic ball on which $u$ is positive and assume that $R<+\infty$. Applying Theorem 2.5 we obtain a contradiction showing the positivity of $\alpha$ on $[0,+\infty)$. 


\section{REFERENCES}

[B] T. Bhattacharya, A nonexistence result for the n-Laplacian, Pacific J . Math. 160 (1993), 19-26 MR 94f:35043

[C-L] K. S. Cheng and J. T. Lin, On the elliptic equations $\Delta u=K(x) u^{\sigma}$ and $\Delta u=K(x) e^{2 u}$, Trans. Amer. Math. Soc. 304 (1987), 639-668. MR 88j:35054

[FC-S] D. Fischer-Colbrie and R. Schoen, The structure of complete stable minimal surfaces in 3-manifolds of nonnegative scalar curvature, Comm. Pure Appl. Math. 33 (1980), 199-211. MR 81i:53044

[G-W] R. E. Greene and H.H. Wu, Function theory on manifolds which possess a pole, Lectures Notes in Math., vol. 699, Springer Verlag, New York, 1979. MR 81a:53002

[L] F. H. Lin, On the elliptic equation $D_{i}\left[a_{i j}(x) D_{j} U\right]-k(x) U+K(x) U^{p}=0$, Proc. Amer. Math. Soc. 95 (1985), 219-226. MR 86k:35041

[K-W] J. L. Kazdan and F. N. Warner, Curvature functions for open 2-manifolds, Ann. of Math. 99 (1974), 203-219. MR 49:7950

[N] W. M. Ni, On the elliptic equation $\Delta u+K(x) u^{\frac{m+2}{m-2}}=0$, its generalizations and applications to geometry, Indiana Univ. Math. J. 31 (1982), 493-539. MR 84e:35049

[Na] M. Naito, A note on bounded positive entire solutions of semilinear elliptic equations, Hiroshima Math. J. 14 (1984), 211-214. MR 86d:35047

[P-W] M. H. Protter and H. Weinberger, Maximum principles in differential equations, PrenticeHall, 1967. MR 36:2935

[RRS1] A. Ratto, M. Rigoli, and A. Setti, On the Omori-Yau maximum principle and its applications to differential equations and geometry, J. Funct. Analysis 134 (1995), 486-510. MR 96k:53062

[RRS2] — A uniqueness result in PDE's and parallel mean curvature immersions in Euclidean space, Complex Variables 30 (1996), 221-233. CMP 96:17

[RRV1] A. Ratto, M. Rigoli, L. Véron, Scalar curvature and conformal deformations of noncompact Riemannian manifolds, C.R. Acad. Sci. Paris Sér. I Math. 318 (1994), 665-670 and Math. Z. (to appear). MR 95a:53061

[RRV2] _ Scalar curvature and conformal deformations of hyperbolic space, J. Funct. Analysis 121 (1994), 15-77. MR 95a:53062

B.B. e M.R. Dipartimento di Matematica, Universitá di Milano, Via Saldini, 50, 20133, Milano, Italy

E-mail address: rigoli@vmimat.mat.unimi.it 